\title{
Fractional-order viscoelasticity applied to describe uniaxial stress relaxation of human arteries
}

\author{
Damian Craiem , Francisco J Rojo , Jose Miguel Atienza , \\ Ricardo L Armentano and Gustavo V Guinea \\ Facultad de Ingenieria, Ciencias Exactas y Naturales, Favaloro University, Buenos Aires, \\ Argentina \\ Departamento de Ciencia de Materiales, Universidad Politecnica de Madrid, Madrid, Spain
}

\begin{abstract}
Viscoelastic models can be used to better understand arterial wall mechanics in physiological and pathological conditions. The arterial wall reveals very slow time-dependent decays in uniaxial stress-relaxation experiments, coherent with weak power-law functions. Quasi-linear viscoelastic (QLV) theory was successfully applied to modeling such responses, but an accurate estimation of the reduced relaxation function parameters can be very difficult. In this work, an alternative relaxation function based on fractional calculus theory is proposed to describe stress relaxation experiments in strips cut from healthy human aortas. Stress relaxation $(1 \mathrm{~h})$ was registered at three incremental stress levels. The novel relaxation function with three parameters was integrated into the QLV theory to fit experimental data. It was based in a modified Voigt model, including a fractional element of order $a$, called spring-pot. The stressrelaxation prediction was accurate and fast. Sensitivity plots for each parameter presented a minimum near their optimal values. Least-squares errors remained below 2\%. Values of order $a=0.1-0.3$ confirmed a predominant elastic behavior. The other two parameters of the model can be associated to elastic and viscous constants that explain the time course of the observed relaxation function. The fractional-order model integrated into the QLV theory proved to capture the essential features of the arterial wall mechanical response.
\end{abstract}

\section{Introduction}

Understanding the changes in the viscoelastic parameters of arteries and their relationships with material properties and structure can provide valuable insight into the functional behavior of the tissue (Armentano et al 2006). Recently, we demonstrated that in certain specific applications where cryografts/prosthesis implants are evaluated, information on viscoelastic 
properties becomes essential to improve patency (Bia et al 2007). Accordingly, biomechanical models are needed to classify vessels and grafts for transplant purposes, aiming to reduce the viscoelastic mismatch observed after the arterial replacement.

To describe the mechanical response of artery walls in vitro, uniaxial tensile loading experiments can be conducted (Fung 1981). Typical tensile tests are employed to characterize static mechanical response whereas ramp-and-hold stress-relaxation tests are used to study time-dependent behavior. Cartilage, tendons and other biological soft tissues are relatively insensitive to the strain rate of the loading ramp phase and present a fast initial descent followed by a very slow relaxation (Kwan et al 1993, Jager 2005, Robinson et al 2004, Zatzman et al 1954). These results are coherent with the well-known dynamic experiments of Handung (1952) and Bergel (1961a, 1961b) that show the relatively wide flat frequency response of the complex elastic modulus in arteries.

The quasi-linear viscoelastic (QLV) approach, introduced by Fung (1981), was successfully applied in soft tissues owing to their relative independence in the frequency responses (Abramowitch and Woo 2004, Funk et al 2000, Lynch et al 2003, Sarver et al 2003, Toms et al 2002). QLV theory suggests that stress in a material can be explained as the convolution of a normalized relaxation function $G(t)$ and a non-linear elastic function. Fung proposed a reduced relaxation function that is based on a continuous spectrum assumption with a nearly constant frequency response for a wide range of frequencies. However, the parameters of this reduced function can be difficult to estimate and show low sensitivity during the adaptation process (Abramowitch and Woo 2004, Doehring et al 2004, Kwan et al 1993, Nigul and Nigul 1987).

Mechanical properties of biomaterials are often represented by linear differential equations, developed from physical models, traditionally including ideal springs and dashpots. Specifically in arteries, Kelvin-Voigt models were unable to fit their particular time and frequency responses with a reduced number of elements (Orosz et al 1997, Westerhof and Noordergraaf 1970). The fractional calculus theory allows derivatives of non-integer order. Accordingly, a new type of element called spring-pot can be conceived. This spring-pot element intermediates between a spring and a dashpot using a fractional-order derivative. If in a pure elastic element, stress $(a)$ and strain (e) are related by a constant $E$ and in a pure viscous dashpot with a first-order derivative, a spring-pot introduces the possibility of gradually interpolating between both by varying the derivative order $a(1>a>0)$. Fractional-order models (FOM) were traditionally restricted to studying viscoelastic properties in polymers (Bagley and Torvik 1983, Doi and Edwards 1986, Ferry 1969, Koeller 1984) but they were recently applied in tissue biomechanics (Djordjevic et al 2003, Kiss et al 2004, Suki et al 1994). They proved to be very efficient in matching several orders of magnitude in frequency responses of different materials with a fewer number of elements.

In a recent work, we used an FOM with two spring-pots to successfully describe the arterial wall frequency response in vivo (Craiem and Armentano 2007). The frequency response of arteries, as other soft tissues, shows certain frequency independence with powerlaw shapes that naturally conform FOM predictions (Kiss et al 2004). The particular stressrelaxation decay in arteries is coherent with this flat frequency spectrum.

Merging these ideas, Doehring et al integrated a fractional-order relaxation function to QLV theory to successfully describe the aortic valve cusp biomechanics (Doehring et al 2005). To our knowledge, this methodology was not yet employed to describe arterial wall mechanics.

In this work, we replace Fung's reduced relaxation function with an alternative $G(t)$ based on fractional calculus. The three parameters of the proposed $G(t)$ arebased on a modified Voigt structure with an ideal spring and a spring-pot. The QLV theory is then applied to describe $1 \mathrm{~h}$ stress-relaxation responses at three incremental stress levels in human arteries based on 


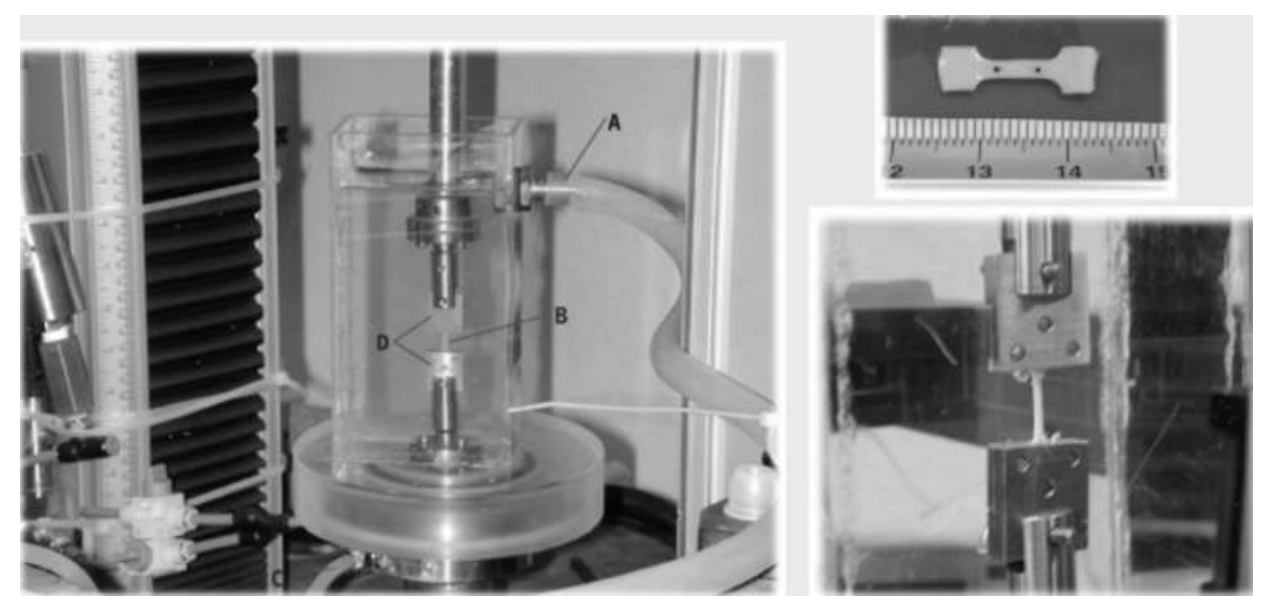

Figure 1. Left: The arterial specimen (B) mounted with two steel fixtures (D) inside a controlled temperature chamber and the tensile testing machine. PBS solution input (C) and output (A). Right-top: human aorta T-bone strip of $2 \mathrm{~mm}$ width and $10 \mathrm{~mm}$ length. Right-bottom: elongation of the specimen during the stress-relaxation experiment.

uniaxial loading experiments. Sensitivity and physical interpretation of the parameters were investigated. Since the novel $G(t)$ is simple and conceptually associated with FOM that show very long relaxation responses with a steep initial decrease, we hypothesized that the QLV theory will straightforwardly fit experimental data in arteries.

\section{Materials and methods}

\subsection{Experimental methods}

Ascending aortic segments were harvested from four human donors (three men and one woman aged 42-51) deceased from causes not related to atherosclerosis. All vessel samples were obtained after acquiring the permission required by current legislation and according to a protocol established and approved by the Ethics Committee of the Hospital Puerta de Hierro in Madrid. Segments were cut out, immediately frozen and stored at $-80{ }^{\circ} \mathrm{C}$. Tests were conducted between two and ten days after excision. Prior to mechanical testing, frozen samples were thawed at room temperature $\left(20^{\circ} \mathrm{C}\right)$.

One representative specimen was extracted from each donor. Each specimen consisted of a circumferentially oriented T-bone strip of nominally $2 \mathrm{~mm}$ width and $10 \mathrm{~mm}$ length dissected using a custom-made steel cutting block (see figure 1). In vivo diameter ranged from 24 to $35 \mathrm{~mm}$ and specimen thickness from 2.00 to $2.25 \mathrm{~mm}$. Details of experimental devices are described elsewhere (Atienza et al 2007). Briefly, two stainless steel fixtures joined the arterial segments to the grips of an electromechanical tensile testing machine (Instron 4411) equipped with a $10 \mathrm{~N}$ load cell. Specimens were enclosed in a PMMA transparent chamber and submerged in PBS solution heated by a thermostatic bath (Unitronic 6320200). The vessel's temperature was $37{ }^{\circ} \mathrm{C}$ and controlled to $0.5^{\circ} \mathrm{C}$ by a K-type thermocouple located in the chamber and close to the artery $(<4 \mathrm{~mm})$. The elongation was measured by the machine's transducer, which gives a precision of $0.001 \mathrm{~mm}$. 


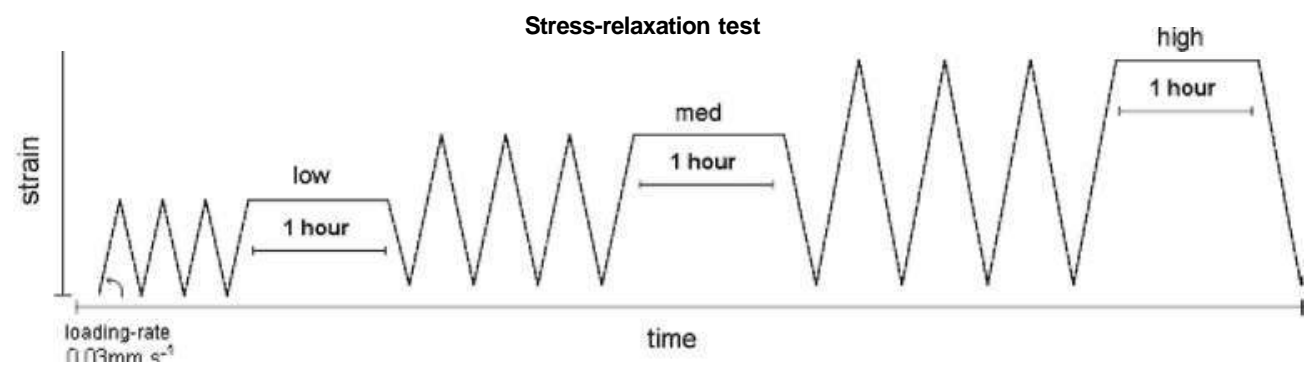

Figure 2. Schematic protocol with three stress levels: low (0.025 MPa), med (0.05 MPa) and high $(0.1 \mathrm{MPa})$. Relaxation time $=1 \mathrm{~h}$. Loading rate during preconditioning ramps: $0.03 \mathrm{~mm} \mathrm{~s}^{-1}$.

Uniaxial stress-relaxation tests were conducted following the protocol with three stress levels outlined in figure 2. In all cases, three preconditioning cycles that preceded the 1-h relaxation phases were matched to low $(0.025 \mathrm{MPa})$, med $(0.05 \mathrm{MPa})$ and high $(0.1 \mathrm{MPa})$ stress levels. Stress levels were selected to match in vivo physiological ranges $(80$ $120 \mathrm{mmHg}$ ), assuming typical values of $25 \mathrm{~mm}$ and $2 \mathrm{~mm}$ for the aorta diameter and thickness, respectively, and maximal values were limited to ensure the integrity of the specimens. The loading and unloading rates were set at $0.03 \mathrm{~mm} \mathrm{~s}^{-1}$ in all cases. Data from ramps and 1-h stress relaxation portions were acquired at a sampling rate of $10 \mathrm{~Hz}$ and reduced to $0.5 \mathrm{~Hz}$ using a decimation function based on an eighth-order low-pass Chebyshev Type I filter (decimate Matlab® function).

Zero-stress extension $\left(\mathrm{L}_{0}\right)$ was defined as the length at which a test specimen first straightened and began to offer measurable tensile resistance. Tensile stretching was defined as an elongation over $\mathrm{L}_{0}$ as $\mathrm{A} .\left(0=L(t) / L_{0}\right.$. Strain was calculated as the incremental elongation with respect to zero-stress extension:

$$
\boldsymbol{e}(\boldsymbol{t})=\underline{\underline{L}(t)-L_{0}} .
$$

Assuming a constant volume, true stress was calculated in $\mathrm{MPa}$ as

$$
a\{t)=\frac{F}{\mathrm{Jo}_{\mathrm{o}}} X(t),
$$

where $F$ is the measured load and So the initial cross-sectional area of the specimen.

\subsection{Constitutive models and parameter estimation}

According to the QLV theory and assuming a zero initial stress state, stress relaxation in a tissue can be calculated as the convolution of a normalized relaxation function $G(t)$ and the non-linear instantaneous elastic response $a^{e}(\mathrm{e})$ as

$$
a\{e(t), t)=G(t)^{*} a^{e}\{e)=1 \quad G(t-r)-^{\wedge}-3 r,
$$

where $e(t)$ is the time-dependent strain, * is the time convolution operator and $\mathrm{G}(\mathrm{f})$ is the relaxation function normalized by the stress at the time of the step input of strain, such that $\mathrm{G}\left(0^{+}\right)=1$.

Preserving Fung's approach, we propose the following three-parameter relaxation function:

$$
G(t)=C+D r^{2}
$$


where the constant $C$ determines the asymptotic response toward the equilibrium and the descending curve is governed by the constant $D$ and the order $a$. This function was not arbitrarily chosen. In a spring with elastic constant $E$ and in a dashpot with viscous constant $r$ ), stress and strain are related with integer-order derivatives, as described in the left-hand side of equation (3):

$$
\begin{aligned}
& \text { spring: } \sigma(t)=E \frac{\mathrm{d}^{0} \varepsilon(t)}{\mathrm{d} t^{0}} \quad \text { spring }- \text { pot: } \sigma(t)=\eta \frac{\mathrm{d}^{\alpha} \varepsilon(t)}{\mathrm{d} t^{\alpha}} . \\
& \text { dashpot: } \sigma(t)=\eta \frac{\mathrm{d}^{1} \varepsilon(t)}{\Delta \cdot 1}
\end{aligned}
$$

Differential operators are not restricted to integer orders. The fractional-order derivative $\alpha$ of a function $f(t)$ can be expressed following the classical definition attributed to Riemann and Liouville as

$$
\begin{gathered}
\frac{\mathrm{d}^{\alpha *} f(t)}{\mathrm{d} t^{\alpha}}=\frac{1}{\Gamma(1-\alpha)} \frac{\mathrm{d}}{\mathrm{d} t} \int_{0}^{t} \frac{f(\theta)}{(t-\theta)^{\alpha}} \mathrm{d} \theta, \\
d t^{a} \\
\mathrm{r}(1-a)<l t \quad J o \quad(t-0)^{a}
\end{gathered}
$$

where $r$ is the Euler gamma function. A spring-pot intermediate element can then be conceived as presented in the right-hand side of equation (3). If in a Voigt model with a spring (elastic constant $E$ ) and a dashpot, the latter is replaced with a spring-pot, stress-relaxation response to a step strain $g\{t)$ of this modified Voigt-FOM results (Magin 2004):

$$
g(t)=E+\frac{n}{\mathrm{r}(1-a)}{ }^{a} .
$$

The parameters of the proposed relaxation function in equation (2) can be associated with the constants in equation (4) to help in the interpretation of the results.

To complete the QLV convolution in equation (1), an elastic function must be selected. Considering other reports (Doehring et al 2004, 2005) and assuming arteries have non-linear exponential stress-strain responses, we propose:

$$
a^{s}(e)=A\left(e^{B s}-1\right) \text {, }
$$

where the parameters $A$ and $B$ must be determined with experimental data.

Data from stress measurements in the experiments consist of two stages: a loading ramp followed by the actual stress relaxation when the strain is held constant. For estimation of the five parameters $(\mathrm{A}, \mathrm{B}, \mathrm{C}, \mathrm{D}, a)$, we minimized the error between the model stress and the measured true stress data, including both stages. The curve-fitting problem was solved in the least-square sense using Matlab® Iqscurvefit function based on the Levenberg-Marquardt algorithm and the convolution in equation (1) using numerical integration with quad Matlab® function. As the relaxation function in equation (2) has a singularity at $t=0$, we compute the convolution from the smallest positive time based on the sampling rate. Before each fitting process, initial values of $A$ and $B$ from the non-linear elastic function in equation (5) were estimated by fitting the ramping phase to each stress-strain curve. Preconditioning was ensured to eliminate hysteretic responses, mostly noticeable in the first or second loop. During the relaxation stage, when the parameters $C, D$ and $a$ were adjusted, $A$ and $B$ were initially allowed to vary only $10 \%$ from their initial values to accelerate the process of fitting. During the adaptation process, we verified that extreme values for $A$ and $B$ were not attained. If this occurred, the $10 \%$ constraint was widened in steps of $10 \%$ until a proper adaptation was achieved.

Initial conditions for the three parameters of the relaxation function in equation (2) and its restraints during the fitting process are explained hereafter. Observing equation (2), the proposed $G(t)$ cannot be normalized to its initial value so as to impose $G\left(0^{+}\right)=1$. Then, the relaxation function was normalized to the asymptotic stress at equilibrium. Initial values for 
$C$ in equation (2) were estimated by dividing the stress averaged during the last 5 min of each relaxation by peak stress (CTMAX) registered just after the loading ramp. As no stress values can exceed CTMAX, constant $C$ was restricted to remain below unity during the fitting process.

In all cases, constant $D$ was given an initial value of 0.5 and restricted to be positive because it is associated with a viscous constant (see equation (4)). The fractional order $a$ was initiated at 0.5 and restricted to remain positive and below unity to represent a spring-pot that interpolated between a pure elastic/viscous element.

To evaluate the quality of fitting, percentage least-squares errors (LSE) relative to the measured values were calculated as

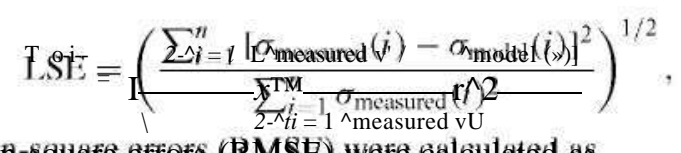

and root-mean=square efrors (RMSE) were calculated as

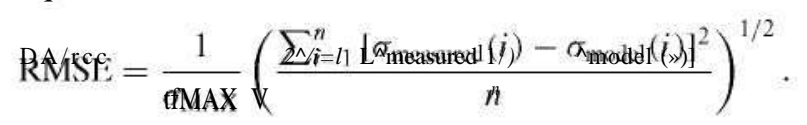

Finally, we analyzed the sensitivity of the three parameters in equation (2). To that end, we calculated the RMSE when two parameters were held constant, and the third parameter was varied around the optimal value. We repeated this methodology to reconstruct sensitivity plots for each parameter.

\subsection{Statistical analysis}

Mean and SD values were calculated for each viscoelastic parameter grouped in three stress levels. Comparisons between groups were addressed by ANOVA and multiple comparisons were performed with the Tukey-Kramer test (JMP software for the Apple Macintosh; SAS Institute, Cary, NC).

\section{Results}

True stress versus strain during the loading ramp and relaxation are shown in figure 3 for each specimen and during three stress levels described in the figure 2 protocol. In all cases, the stress-strain curves tended to describe a typical J-shape with pronounced non-linearity for higher distensions.

A representative example of the model fitting accuracy is shown in figure 4 . The predicted stress closely followed the measured data during the ramp and the relaxation portions of the curve. The algorithm was fast with a mean adaptation time of $20 \mathrm{~s}$ ( $<200$ iterations). The point-wise difference error was also calculated and shown to be more significant during the last portion of the ramping part (see ERROR in figure 4). The five adjusted parameters of the model are presented in table 1 with calculated errors as defined by equations (6) and (7). For every specimen and all the stress levels, errors were below $2 \%$. Error magnitude did not depend on stress levels. The parameters of the resulting relaxation function followed different behaviors with incremental stress levels (see table 1). The constant $C$ tended to remain stable from low to med stress levels and to diminish during high stress $(p<0.05)$. The constant $D$ tended to increase with stress $(p<0.05$ for high with respect to low) whereas the fractional order $a$ ranged between 0.1 and $0.3(p=$ NS). The elastic function described in (5) has two parameters. The proportional constant $A$ tended to decrease and the exponent constant $B$ tended to increase with increasing stress levels, attaining significant differences only for high levels with respect to low $(p<0.05)$. 

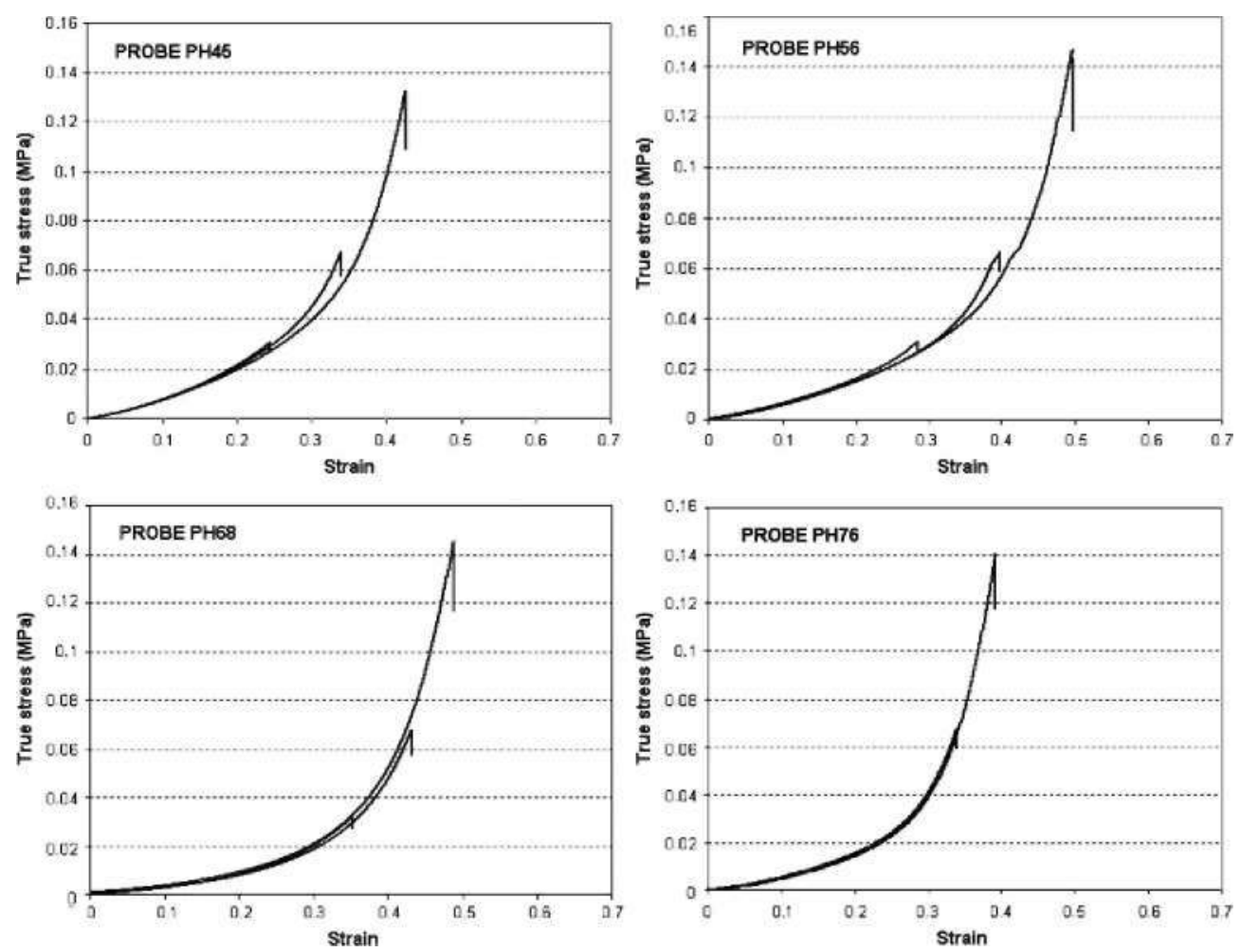

Figure 3. True stress versus strain responses to the stress-relaxation protocol for all specimens.

Table 1. Estimated viscoelastic parameters. LOW, MED and HIGH are the stress levels defined as $0.025 \mathrm{MPa}, 0.05 \mathrm{MPa}$ and $0.1 \mathrm{MPa}$, respectively.

\begin{tabular}{llllllll}
\hline Stress & $A(\mathrm{KPa})$ & $B$ & $C$ & $D\left(\mathrm{~s}^{\mathrm{a}}{ }^{\mathrm{a}}\right)$ & $a$ & LSE $\left(10 \sim^{3}\right)$ & RMSE $\left(10 \sim^{3}\right)$ \\
\hline LOW & $6.30 \pm 3.0$ & $6.23 \pm 1.2$ & $0.897 \pm 0.02$ & $0.309 \pm 0.11$ & $0.205 \pm 0.05$ & $8.6 \pm 0.6$ & $2.4 \pm 0.2$ \\
MED & $3.22 \pm 2.1$ & $8.23 \pm 1.7$ & $0.868 \pm 0.03$ & $0.436 \pm 0.09$ & $0.124 \pm 0.03$ & $9.8 \pm 2.0$ & $5.7 \pm 1.1$ \\
HIGH & $1.75 \pm 1.0^{\mathrm{a}}$ & $9.87 \pm 1.8^{\mathrm{a}}$ & $0.793 \pm 0.04^{\text {arb }}$ & $0.603 \pm 0.10^{\mathrm{a}}$ & $0.186 \pm 0.06$ & $1.4 \pm 1.0$ & $1.6 \pm 1.3$ \\
\hline
\end{tabular}

Parameters $A$ and $B$ from the elastic function are defined in equation (5) and parameters $C, D$ and $a$ from the relaxation function in equation (2). LSE and RMSE are fitting errors calculated with equations (6) and (7). ${ }^{\mathrm{a}} \mathrm{P}<0.05$ with respect to LOW stress level. ${ }^{\mathrm{b}} \mathrm{P}<0.05$ with respect to MED stress level (Tukey-Kramer HSD test).

In the sensitivity analysis of the relaxation function parameters, minimum errors were clearly visible in every test, as shown in figure 5 for a representative case. All three parameters during every stress level found their optimal value, with a single minimum, in the least-squares sense.

\section{Discussion}

In this work, we applied the QLV theory to describe stress relaxation in human aorta in vitro, where the reduced relaxation function proposed by Fung was replaced with an alternative function formulated from a modified Voigt fractional-order model. Three main outcomes should be emphasized. (1) Using the proposed three-parameter relaxation function, the fitting 


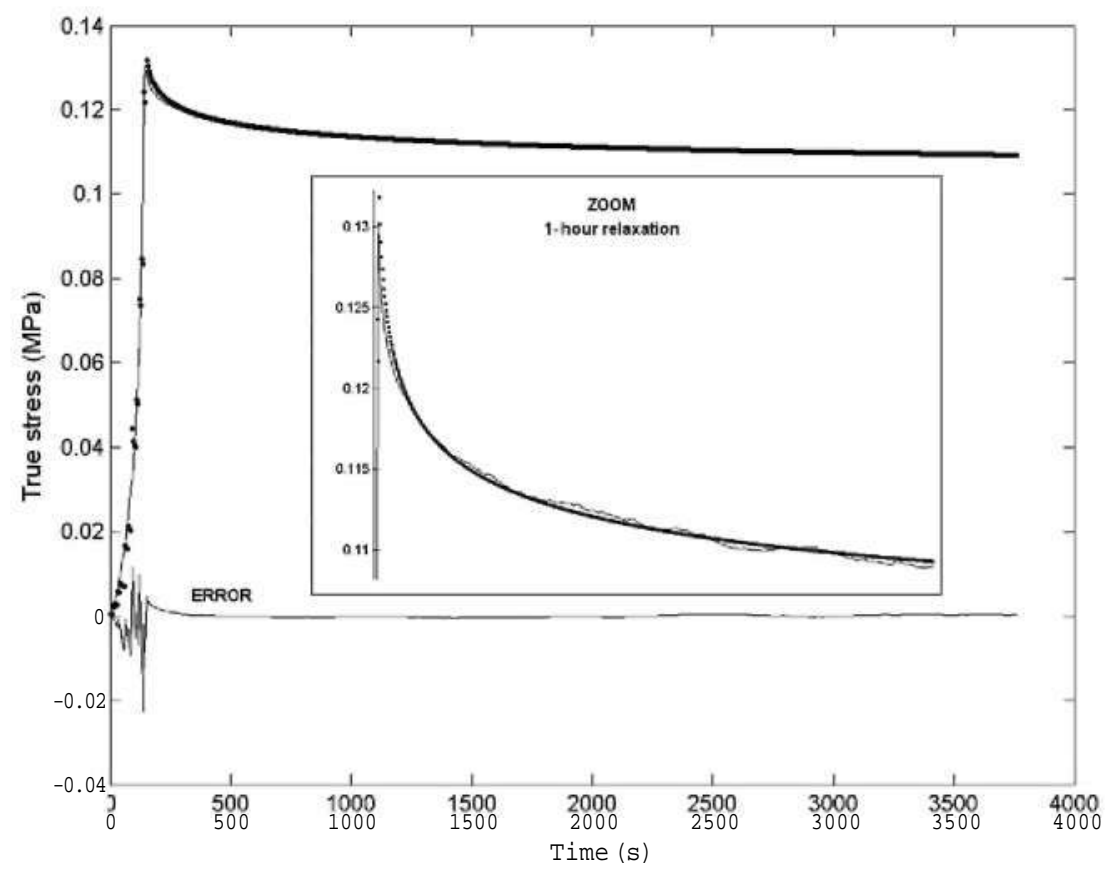

Figure 4. Ramp and stress relaxation example for specimen PH45 during stress level HIGH. Measured data in solid line and model fitting in dots. The difference (ERROR) between them is also drawn as a solid line. A zoomed area of the peak and relaxation portions is showed in the center of the figure.

process resulted fast for «al800 stress values in each experimental data set. (2) The time course predicted by the model conformed power-law functions that properly followed stress relaxation during $1 \mathrm{~h}$ in three increasing true-stress levels. Least-squares errors remained below 2\%. (iii) All parameters were sensitive in the least-squares sense. A unique minimum value was found around optimal values, (iv) The constants of the proposed relaxation function can help to identify arterial elastic and viscous behavior.

In this study, we found that stress relaxation in human arteries presented a rapid initial decay of force, followed by decreasing rate of decay and asymptotic approach to G(oo). Our results are similar to others found in carotid dog arteries (Zatzman et al 1954), myocardium tissue (Miller et al 1997) and other organic materials (Jager 2005). There are no other studies to directly compare QLV results in human aorta of healthy donors, probably due to the arterial procurement complexity. Nevertheless, there is a general consent that supports the idea of non-exponential stress relaxation curves in arteries (Kalita and Schaefer 2008).

Curve-fitting methods with Kelvin-Voigt models need several elements to accurately fit experimental data (Orosz et al 1997, Westerhof and Noordergraaf 1970). More important, unlike the linear viscoelastic modeling, QLV is able to capture the non-linearity in elasticity. The classic QLV reduced relaxation function has three parameters including a viscoelastic constant, a short and a long time constant. However, using Fung's relaxation function, parameters estimation and sensitivities remain difficult (Abramowitch and Woo 2004, Doehring et al 2005, Kwan et al 1993, Miller et al 1997). In this work, a simple relaxation function based on an FOM was proposed in equation (2) with three parameters, which showed accurate and fast adaptations for long $(1 \mathrm{~h})$ stress-relaxation tests (see the center of 


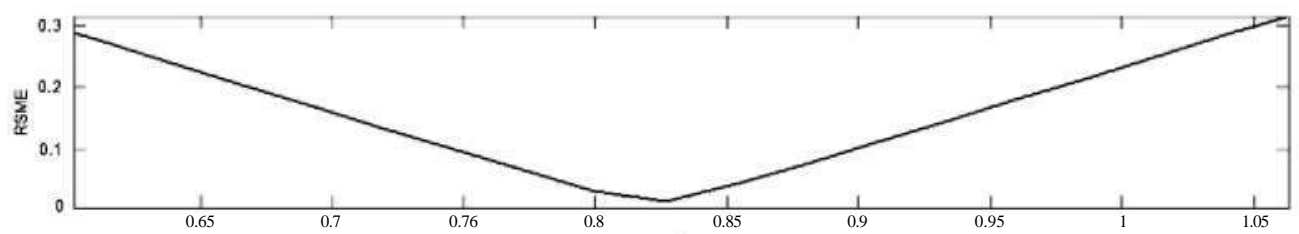

c
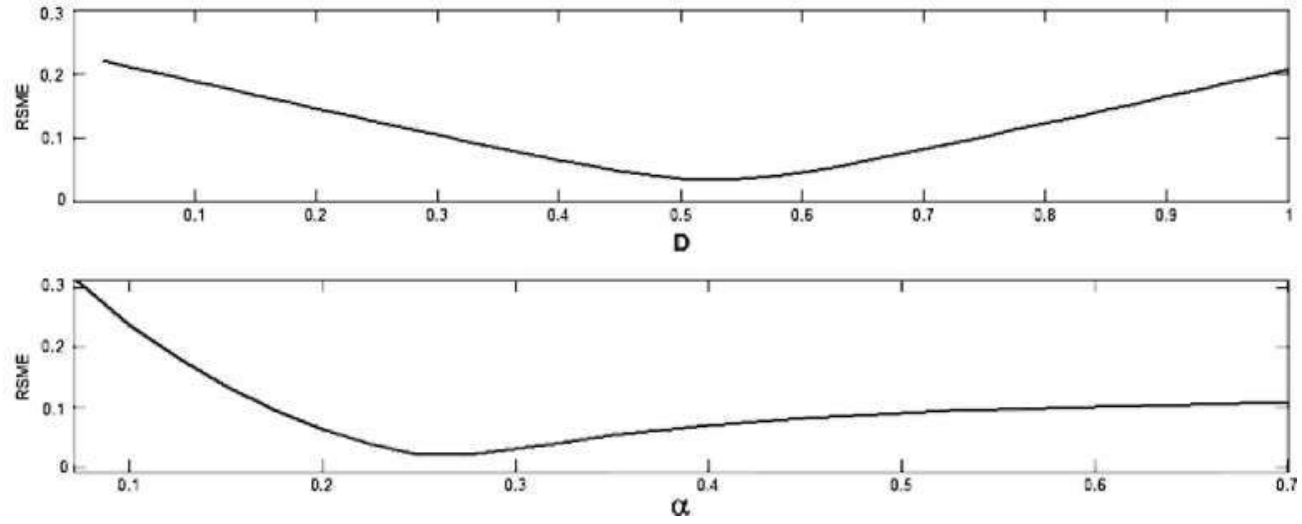

Figure 5. Representative case for the sensitivity analysis in specimen $\mathrm{PH} 76$ during stress level = HIGH experiment. RMSE = root-mean-square error as defined in equation (7) with respect to the parameters C, D and $a$ in the proposed relaxation function in equation (2).

figure 4). Moreover, sensitivity plots for each parameter confirmed a proper optimum value after the fitting process (figure 5). This increase in parameter sensitivity using a fractionalorder function into QLV theory was also reported describing the aortic valve cusp with a similar methodology (Doehring et al 2005). It seems that the natural power-law decay, associated to fractional models, is partly responsible for sensitivity and matching improvement.

With respect to the relaxation function parameters, the alternative of accounting with an elastic and a viscous constant associated with a fractional order should be discussed. In a previous complete mechanical model of arterial wall (Armentano et al 1995), a parallel elastic component was made of one spring, representing elastin, and a number of much stiffer springs of different lengths, associated to collagen. This model of collagen fibers was based on the disconnecting hook model. At very low transmural pressures, the wall stress is borne almost exclusively by elastin. As the fully relaxed vessel is stretched, increasing numbers of stiffer collagen fibers are recruited. Vascular smooth muscle can act as an active element that stretches fibers, modulating viscoelasticity within the arterial wall. We recently showed that this activation mechanism in vivo can be associated to an order modification of a spring pot in a fractional model with three parallel elements (Craiem and Armentano 2007). In the present work, we included a relaxation function derived from a modified Voigt model with one spring and one spring-pot. A second spring-pot was neglected because no active mechanisms are evidenced in vitro. In this sense, the constant $C$ could be associated to a pure elastic response. Constant $D$ in equation (2) could be associated to a pure viscous constant when the order $a$ tends to unity. When the fractional order takes an intermediate value, a viscoelastic effect is attended. Since $a$ resulted around $\ll 0.2$ (table 1 ), a predominant elastic behavior is confirmed. Wall viscosity, represented in the spring-pot element, depends on the arterial structure, partly due to passive contribution of vascular smooth muscle (Armentano et al 2006). Viscosity modulates energy dissipation within the arterial wall and has a beneficial 
effect reducing vessel fatigue (Zhang et al 2007). Thus, each element of the three-parameter relaxation function is associated to specific aspects of the arterial wall: the constant $C$ to pure elastic behavior and constants $D$ and $a$ to viscous energy dissipation. The non-linearity in stress-strain curves is mostly contemplated by the elastic function with constants $A$ and $B$.

With respect to fractional orders, we found values of $a$ in the range 0.1-0.3 similar to the aortic valve tissue (Doehring et al 2005), canine liver tissue (Kiss et al 2004) and lung tissue (Suki et al 1994). These values support the idea of a fractal-like structure (Magin 2004). As other collagenous tissues, arteries are arranged hierarchically and fractional models seem to naturally adapt to this kind of structure (Doi and Edwards 1986, Ferry 1969, Schiessel and Blumen 1995). At the moment, mathematical demonstrations connecting fractional models with material structure have been reported in polymers (Bagley and Torvik 1983), although some hypothesis including biological tissues and cell rheology were presented (Djordjevic e? a/2003, Sukie? a/1994).

We also found that for higher stress levels, the constant $C$ decreased and the constant $D$ increased. Constant $C$ is related to the equilibrium stress relaxation that is reported to decrease with higher strains (Sarver et al 2003). With respect to the constant $D$ increase, it might indicate an enhancement in energy dissipation due to stronger solicitations. It remains to be determined whether this relaxation response is intrinsically stress-dependent (Abramowitch and Woo 2004). Also, while the material stretched in each higher elongation, some structural molecular configurations could have changed, partially modifying viscoelastic parameters. Nevertheless, these changes were small, observing the superimposed stress-strain curves in figure 3. Evidently, higher elongations may produce alterations in the molecular structure of the tested materials with progressive stretching. In view of these results, further measurement protocols with alternative preconditioning phases should be tested to better understand stressdependence in arterial wall segments.

This work also revealed some limitations. Regarding the fitting algorithm, some algorithmic concerns were expressed when fractional functions were included in parameter estimation (Doehring et al 2004, Miller et al 1997). However, all the parameters proved sensitive and clear minimums were found in a least-squares error sense (figure 5). Moreover, the simplicity of the relaxation function in equation (4) helped the algorithm to quickly converge to stable values even with very long relaxation experiments. The experiments in this work were conducted on four specimens harvested from human arteries. This is partly due to the complex process that involves working with fresh human arteries from healthy donors. Uniaxial experiments are simple and then broadly applied to describe soft tissue biomechanics, but arteries are known to be anisotropic. Multidimensional studies, which employ finiteelement models, partially overcome this limitation but usually increase computational effort (Holzapfel 2006). The presented framework aimed to prove that fractional models can be used to describe mechanical responses in arteries whereas new models should extend these ideas to multiple dimensions.

\section{Conclusion}

An alternative relaxation function based on a modified Voigt-FOM was integrated into the QLV theory to describe stress relaxation in human aorta. The model predicted a power-law decay that matched the time course of all stress relaxation experimental data. Using this simplified relaxation function, the adaptation algorithm resulted to be fast and accurate. In the VoigtFOM model, the spring can be associated with the asymptotic pure elastic response whereas the spring-pot with two parameters modulates the viscoelastic behavior, responsible for energy 
dissipation within the arterial wall. Further experiments in time and frequency domains should be scheduled to study stress relaxation during vascular smooth muscle activation.

\section{Acknowledgments}

The authors gratefully acknowledge financial support for this work provided by the Universidad Politecnica de Madrid, Spain, through projects AL07-PID-016, AL08-PID-014 and AL08RT03, the Ministerio de Ciencia y Tecnologia through project MAT 2005-06320 and by the Comunidadde Madrid through programs ESTRUMAT/S-0505/MAT/000077 and MADR.IBCM/SSAL/031/2006. This work was also partly supported by BID 1201/OC-AR/PICT \# 14334 of the Secretaria de Ciencia, Tecnologia e Innovation Productiva, Argentina. Help and useful comments from Dr Carlos C Garcia-Montero, Dr Raul Burgos and Dr Patrice Flaud (ECOS-Sud Argentina/France no. A06S02) are also acknowledged.

\section{References}

Abramowitch S D and Woo S L 2004 An improved method to analyze the stress relaxation of ligaments following a finite ramp time based on the quasi-linear viscoelastic theory J. Biomech. Eng. 126 92-7

Armentano R L, Barra J G, Levenson J, Simon A and Pichel R H 1995 Arterial wall mechanics in conscious dogs. Assessment of viscous, inertial, and elastic moduli to characterize aortic wall behavior Circ. Res. 76 468-78

Armentano R L, Barra J G, Santana D B, Pessana F M, Graf S, Craiem D, Brandani L M, Baglivo H P and Sanchez R A 2006 Smart damping modulation of carotid wall energetics in human hypertension: effects of angiotensin-converting enzyme inhibition Hypertension 47 384-90

Atienza J M, Guinea G V, Rojo F J, Burgos R J, Garcia-Montero C, Goicolea F J, Aragoncillo P and Elices M 2007 The influence of pressure and temperature on the behavior of the human aorta and carotid arteries Rev. Esp. Cardiol. 60 259-67

Bagley R L and Torvik P J 1983 A theoretical basis for the application of fractional calculus to viscoelasticity J. Rheol. 27 201-10

Bergel D H 1961a The dynamic elastic properties of the arterial wall J. Physiol. 156 458-69

Bergel D H 1961b The static elastic properties of the arterial wall J. Physiol. 156 445-57

Bia D, Zocalo Y, Pessana F, Armentano R, Perez H, Saldias M and Alvarez I 2007 Differential functional coupling between human saphenous cryoallografts and arteries: importance of the arterial type and the biomechanical parameter evaluated Artif. Organs 31 809-18

Craiem D and Armentano R L 2007 A fractional derivative model to describe arterial viscoelasticity Biorheology 44 251-63

Djordjevic V D, Jaric J, Fabry B, Fredberg J J and Stamenovic D 2003 Fractional derivatives embody essential features of cell rheological behavior Ann. Biomed. Eng. 31 692-9

Doehring T C, Carew E O and Vesely I 2004 The effect of strain rate on the viscoelastic response of aortic valve tissue: a direct-fit approach Ann. Biomed. Eng. 32 223-32

Doehring T C, Freed A D, Carew E O and Vesely I 2005 Fractional order viscoelasticity of the aortic valve cusp: an alternative to quasilinear viscoelasticity J. Biomech. Eng. 127 700-8

Doi M and Edwards S F 1986 The Theory of Polymer Dynamics (Oxford: Clarendon)

Ferry J D 1969 Viscoelastic Properties of Polymers (New York: Wiley) pp 195-291

Fung Y C 1981 Biomechanics: Mechanical Properties of Living Tissues (Berlin: Springer)

Funk J R, Hall G W, Crandall J R and Pilkey W D 2000 Linear and quasi-linear viscoelastic characterization of ankle ligaments J. Biomech. Eng. 122 15-22

Hardung V 1952 Method for measurement of dynamic elasticity and viscosity of caoutchouc-like bodies, especially of blood vessels and other elastic tissues Helv. Physiol. Pharmacol. Acta 10 482-98

Holzapfel G A 2006 Determination of material models for arterial walls from uniaxial extension tests and histological structure /. Theor. Biol. 238 290-302

Jager I L 2005 Viscoelastic behavior of organic materials: consequences of a logarithmic dependence of force on strain rate J. Biomech. 38 1451-8

Kalita P and Schaefer R 2008 Mechanical models of artery walls Arch. Comput. Methods Eng. 15 1-36

Kiss M Z, Varghese T and Hall T J 2004 Viscoelastic characterization of in vitro canine tissue Phys. Med. Biol. 49 4207-18 
Koeller R C 1984 Applications of fractional calculus to the theory of viscoelasticity J. Appl. Mech. 51 299-307

Kwan M K, Lin T H and Woo S L 1993 On the viscoelastic properties of the anteromedial bundle of the anterior cruciate ligament $J$. Biomech. 26 447-52

Lynch H A, Johannessen W, Wu J P, Jawa A and Elliott D M 2003 Effect of fiber orientation and strain rate on the nonlinear uniaxial tensile material properties of tendon J. Biomech. Eng. 125 726-31

Magin R L 2004 Fractional calculus in bioengineering, part 2 Crit. Rev. Biomed. Eng. 32 105-93

Miller C E, Vanni M A and Keller B B 1997 Characterization of passive embryonic myocardium by quasi-linear viscoelasticity theory J. Biomech. 30 985-8

Nigul I and Nigul U 1987 On algorithms of evaluation of Fung's relaxation function parameters J. Biomech. 20 343-52

Orosz M, Molnarka G and Monos E 1997 Curve fitting methods and mechanical models for identification of viscoelastic parameters of vascular wall-a comparative study Mon. Sci. Monit. 3 599-604

Robinson P S, Lin T W, Reynolds P R, Derwin K A, Iozzo R V and Soslowsky L J 2004 Strain-rate sensitive mechanical properties of tendon fascicles from mice with genetically engineered alterations in collagen and decorin J. Biomech. Eng. 126 252-7

Sarver J J, Robinson P S and Elliott D M 2003 Methods for quasi-linear viscoelastic modeling of soft tissue: application to incremental stress-relaxation experiments J. Biomech. Eng. 125 754-8

Schiessel H and Blumen A 1995 Mesoscopic of the sol-gel transition: ladder models and fractal networks Macromolecules 28 4013-9

Suki B, Barabasi A L and Lutchen K R 1994 Lung tissue viscoelasticity: a mathematical framework and its molecular basis /. Appl. Physiol. 76 2749-59

Toms S R, Dakin G J, Lemons J E and Eberhardt A W 2002 Quasi-linear viscoelastic behavior of the human periodontal ligament/. Biomech. 35 1411-5

Westerhof N and Noordergraaf A 1970 Arterial viscoelasticity: a generalized model. Effect on input impedance and wave travel in the systematic tree J. Biomech. 3 357-79

Zatzman M, Stacy R W, Randall J and Eberstein A 1954 Time course of stress relaxation in isolated arterial segments Am. J. Physiol. Ill 299-302

Zhang W, Liu Y and Kassab G S 2007 Viscoelasticity reduces the dynamic stresses and strains in the vessel wall: implications for vessel fatigue Am. J. Physiol. Heart Circ. Physiol. 293 2355-60 